\title{
Glaucoma maligno durante realização de curva tensional diária com tonômetro de contorno dinâmico
}

\section{Malignant glaucoma during execution of diurnal curve of intraocular pressure with dynamic contour tonometer}

Marcos Antônio Ferraz Rego', Francisco Eduardo Lopes Lima², Marcos Pereira de Ávila ${ }^{3}$

\section{ResUMO}

O glaucoma maligno é uma doença caracterizada por pressão intraocular elevada com câmara anterior rasa na presença de iridectomia periférica patente. Na sua forma clássica, o glaucoma maligno é raro, e representa uma das mais sérias complicações da cirurgia fistulizante em pacientes com ângulo estreito ou glaucoma de ângulo fechado. O presente trabalho relata um caso de uma paciente do sexo feminino que desenvolveu crise de glaucoma por bloqueio ciliar (maligno) precipitada pela realização de curva tensional diária de pressão intraocular com tonômetro de contorno dinâmico.

Descritores: Glaucoma de ângulo fechado; Tonometria ocular; Tomografia de coerência óptica; Lasers de estado solido; Relatos de casos

\begin{abstract}
Malignant glaucoma is an entity characterized by intraocular pressure (IOP) with a shallow anterior chamber in the presence of a patent peripheral iridectomy. In its classic form, malignant glaucoma is rare but one of the most serious complications of fistulizing surgery in patients with narrow angle or angle closure glaucoma. In this case we have a female patient who developed crisis of ciliary block glaucoma (malignant) during performance of circadian IOP curve with dynamic contour tonometer.
\end{abstract}

Keywords: Glaucoma, angle-closure; Tonometry, ocular; Tomography, optical coherence; Lasers, solid-state; Case reports

${ }^{1}$ Centro Brasileiro da Visão - Brasília (DF), Brasil;

${ }^{2}$ Universidade Federal de Goiás (UFG) - Goiânia (GO), Brasil;

${ }^{3}$ Universidade Federal de Goiás (UFG) - Goiânia (GO), Brasil.

Os autores declaram inexistir conflitos de interesse

Recebido para publicação em 20/7/2011 - Aceito para publicação em 11/12/2011 


\section{INTRODUÇÃO}

$\mathbf{0}$ glaucoma maligno (GM) foi descrito pela primei-ra vez por Von Graefe em 1869, e refere-se a um estreitamento da câmara anterior acompanhado de aumento progressivo e sustentado da pressão intraocular (PIO), na presença de uma iridectomia periférica patente, sendo uma rara complicação cirúrgica $^{(1)}$. O termo GM reflete a dificuldade no tratamento e, frequentemente, um pobre resultado após terapia convencional na época em que foi descrito. Olhos com câmara anterior rasa, raio de curvatura menor e diâmetro anteroposterior do globo ocular reduzido apresentam risco de sofrer desta condição, que acontece de uma maneira geral, após cirurgia intraocular. Essa predisposição anatômica pode ser devida à aposição dos processos ciliares à lente intraocular ou ao vítreo anterior, o que causaria desvio do aquoso para dentro do humor vítreo originando o termo glaucoma por bloqueio ciliar ${ }^{(2)}$. $\mathrm{O}$ entendimento moderno da patogênese desta condição sugere que uma hialóide anterior anormal e impermeável também esteja envolvida ${ }^{(3)}$. O evento que estimula a formação deste raro glaucoma é a incapacidade de gerar fluxo de humor aquoso através do vítreo o suficiente para compensar a drenagem deste para a câmara anterior, submetido a maior nível pressórico, gerado pela expansão da coróide. Existe maior probabilidade de ocorrência em um olho com resistência aumentada para fluxo de humor aquoso no vítreo. Desta forma, é possível que a limitação de fluxo de humor aquoso através da hialóide possa contribuir para fechamento angular primário em olhos com outros fatores de risco para essa doença, e poderia ser o principal fator predisponente para glaucoma maligno nos olhos de tamanho normal ${ }^{(4)}$. O desvio do aquoso para dentro do vítreo resulta em expansão do volume do vítreo que secundariamente empurra a lente e a íris para a córnea. Isso resulta em uma das marcas desta condição: profundidade da câmara anterior rasa (distância córneo-lenticular) juntamente com a periferia (distância íris-córnea). Em contraste, no glaucoma primário de ângulo fechado, causado por bloqueio pupilar, a profundidade axial permanece normal para o olho, enquanto que a profundidade periférica está rasa.

Este artigo tem o objetivo de expor um caso clínico de uma paciente que desenvolveu crise de GM durante a realização de exame de curva tensional diária com tonômetro de contorno dinâmico (Pascal).

\section{Relato de Caso}

MRF, sexo feminino, 67 anos, buscou atendimento médico no setor de glaucoma no mês de abril de 2011 para avaliação de glaucoma. A paciente apresentava como queixa baixa acuidade visual e sensação de "membrana sobre a visão" do olho esquerdo, além de desconforto neste olho desde a cirurgia de glaucoma realizada há oito meses. A mesma estava em uso de colírios de latanoprost, associação de maleato de timolol $0,5 \%$ com cloridrato de dorzolamida $2 \%$ e tartarato de brimonidina $0,2 \%$ apenas em olho direito e lubrificante ocular (Systane) em ambos os olhos. Refere ter sido submetida à cirurgia de catarata há quatro anos e a trabeculectomia em OE (olho esquerdo) há oito meses.

Ao exame oftalmológico apresentava acuidade visual com melhor correção de 20/50 (-1,50()-1,25x135) em OD e 20/ $25(-3,25()-1,75 \times 100)$ em OE. À biomicroscopia ela apresentou no olho direito (OD): pálpebras sem alterações, pterígio moderado, câmara intermediária, pseudofacia com LIO centrada e transparente. No olho esquerdo (OE) exibiu pálpebras normais, ampola fistulizante difusa com vascularização moderada, córnea normal, câmara rasa, iridectomia pérvia e
LIO (lente intraocular) centrada. A gonioscopia do olho direito mostrava ângulo estreito grau I de Schaeffer com goniossinéquias dispersas e OE com ângulo estreito Grau I de Schaeffer e com goniossinéquias dispersas. A PIO com tonômetro de Goldman era de $24 / 14 \mathrm{mmHg}$ às $15 \mathrm{~h} 54 \mathrm{~min}$. Ao exame de fundo de olho, o OD apresentava relação escavação/disco de 0,8/0,8 com rima inclinada superiormente e CFN (camada de fibras nervosas da retina) diminuída e mácula sem alterações. Em OE detectou-se nervo óptico com relação escavação/disco de 0,9/0,9 com rima inclinada superiormente, CFN diminuída difusamente e mácula normal. Diante do quadro observado, foi levantada a hipótese diagnóstica de pseudofacia associada à glaucoma crônico de ângulo estreito descontrolado em olho direito e glaucoma de ângulo estreito controlado após cirurgia fistulizante em OE. Foram solicitados exames complementares para confirmação diagnóstica, dentre os quais: estéreo fotografia de papila, campimetria computadorizada, paquimetria e curva tensional diária.

Após uma semana, a paciente retornou para realizar exames e a curva tensional diária com tonômetro de contorno dinâmico detectou PIO bastante elevada em OD, sem alteração da profundidade da câmara anterior; houve aumento sustentado da PIO no OE e correspondente estreitamento progressivo da câmara anterior o que foi detectado ao exame biomicroscópico no OE, aposição do complexo irido-lenticular à córnea com atalamia na medida realizada após às $17 \mathrm{~h} 00 \mathrm{~min}$. A CTD mostrou os seguintes resultados às $08 \mathrm{~h} 00 \mathrm{~min}$, $11 \mathrm{~h} 00 \mathrm{~min}, 14 \mathrm{~h} 00 \mathrm{~min}$ e $17 \mathrm{~h} 00 \mathrm{~min}$. OD: 35,$1 ; 31,2 ; 28,3$; e $26,2 \mathrm{mmHg}$; em OE: 12,$1 ; 16,4 ; 26,0$; e $28,1 \mathrm{mmHg}$; foi realizada ainda tonometria de Goldman às $18 \mathrm{~h} 30 \mathrm{~min}$ que evidenciou PIO: 33/ $30 \mathrm{mmHg}$. À biomicroscopia, o OE mostrava hiperemia conjuntival moderada, edema epitelial periférico da córnea, ampola difusa e vascularizada, atalamia, iridectomia ampla superior e LIO centrada.

Foi evidenciado glaucoma crônico de ângulo estreito no OD e glaucoma agudo por bloqueio ciliar (maligno) no OE. Realizouse intervenção imediata com instituição de tratamento clínico específico com inibidores da anidrase carbônica orais, colírio de atropina a $1 \%$ e hipotensores tópicos. No dia seguinte, a PIO permanecia elevada, a câmara atalâmica e a medida do tonômetro de Goldman às $18 \mathrm{~h} 41 \mathrm{~min}$ era de $32 / 30 \mathrm{mmHg}$. Não havendo controle pressórico, foram realizados hialoidotomia e capsulotomia posterior com NDYag laser no OE, que levou ao controle pressórico e estabilização do quadro clínico deste olho. A paciente encontra-se em tratamento clínico com máxima medicação tolerada em $\mathrm{OD}$, pois não deseja se submeter à cirurgia no momento; e encontra-se sem a necessidade de medicações para o olho esquerdo.

Foi realizada tomografia de coerência óptica de segmento anterior em ambos os olhos (Figuras 1 e 2), que mostrou confi-

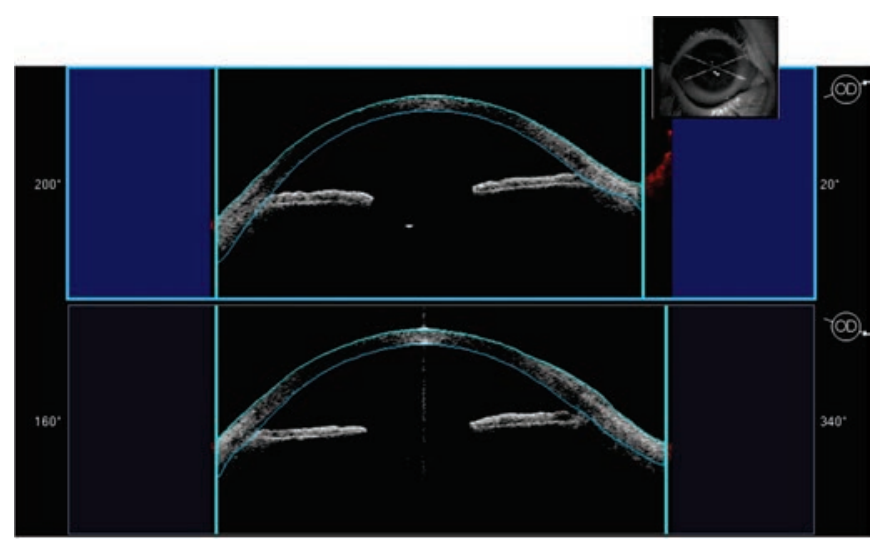

Figura 1: OCT de segmento anterior do OD 


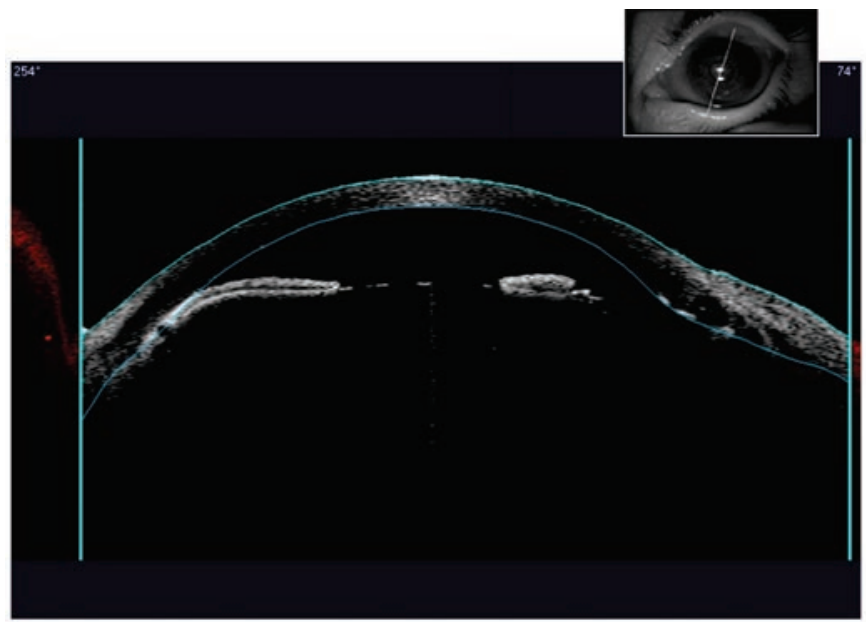

Figura 2: OCT de segmento anterior do OE, mostrando iridectomia pérvia e ampola fistulizante funcionante

guração anatômica da câmara anterior normal olho direito e uma configuração anatômica predisponente ao fechamento angular primário e consequentemente ao glaucoma maligno no olho esquerdo mesmo após realização de hialoidotomia e capsulotomia com Nd-Yag laser.

\section{DiscussÃo}

Diversos artigos têm demonstrado a ocorrência de glaucoma maligno após realização de cirurgia de catarata com implante de $\mathrm{LIO}^{(5-7)}$, iridectomia com Nd-Yag laser ${ }^{(8-11)}$, uso de mióticos ${ }^{(12)}$, ciclofotocoagulação com Nd-Yag laser ${ }^{(13)}$, esclerostomia a laser ${ }^{(14)}$ e após trabeculectomia ${ }^{(15)}$, porém não há relatos prévios de pacientes que tenham desenvolvido crise de glaucoma maligno durante realização de curva tensional diária (CTD). O presente artigo ilustra o comportamento da PIO e da profundidade da câmara anterior de uma paciente que tinha predisposição ao GM e desenvolveu crise durante realização do exame de CTD.

O tonômetro de Aplanação de Goldman é o mais utilizado para a realização do exame de CTD, porém, atualmente o tonômetro de contorno dinâmico (Pascal) tem sido utilizado para esta finalidade por possuir maior independência em relação à espessura e às propriedades biomecânicas da córnea, pois mede a amplitude do pulso ocular e ainda fornece índice de qualidade da medição ${ }^{(16)}$, apesar disso, existe poucos estudos em longo prazo, o que limita sua utilização. De modo diferente do tonômetro de Goldman, o tonômetro de Contorno dinâmico não aplana ou indenta a córnea, ele tem uma superfície de contato côncava que comprime toda a extensão da córnea, o que hipoteticamente pode exercer uma pressão no sentido anteroposterior do bulbo ocular e movimenta a córnea contra o complexo írido-lenticular, predispondo os indivíduos susceptíveis à crise de glaucoma maligno, fato este que necessita de comprovação em estudos mais bem estruturados.

A avaliação através da tomografia de coerência óptica do segmento anterior tem importância no diagnóstico da etiologia do glaucoma, bem como no acompanhamento de sua progressão(17).

O tratamento do glaucoma maligno pseudofácico tem sido conduzido de diversas maneiras, como: ablação a laser dos processos ciliares ${ }^{(18,19)}$, hialoidotomia e capsulotomia posterior com Nd-Yag laser ${ }^{(20-23)}$, e ainda têm sido propostos diversos tratamentos cirúrgicos como vitrectomia anterior via pars plana ${ }^{(24-26)}$. No caso relatado, o tratamento instituído obteve resultado satisfatório com total reversão do glaucoma como demonstra a figura 2.

\section{Conclusão}

O glaucoma maligno é uma doença rara que, apesar de apresentar boa resposta aos tratamentos atuais, frequentemente necessita de tratamento cirúrgico. Enquanto estudos mais elaborados avaliando o risco de crise de glaucoma maligno não sejam conduzidos, devemos atentar ao risco de submeter pacientes portadores de glaucoma de ângulo estreito à realização de CTD com a utilização do tonômetro de contorno dinâmico.

\section{ReFERÊNCIAS}

1. Von Graefe A. Beiträge zur pathologie und therapie des glaucoms. Arch Ophthalmol. 1869;15:108-252.

2. Weiss DI, Shaffer RN. Ciliary block (malignant) glaucoma. Trans Am Acad Ophthalmol Otolaryngol. 1972;76(2):450-61.

3. Epstein DL. The malignant glaucoma syndromes. In: Chandler PA, Epstein DL, editors. Chandler and Grant's glaucoma. 4th ed. Baltimore: Lippincott Williams \& Wilkins; 1996. p. 285-303.

4. Quigley HA, Friedman DS, Congdon NG. Possible mechanisms of primary angle-closure and malignant glaucoma. J Glaucoma. 2003;12(2):167-80. Comment in J Glaucoma. 2003;12(5):446-7; author reply 447-8.

5. Hanish SJ, Lamberg RL, Gordon JM. Malignant glaucoma following cataract extraction and intraocular lens implant. Ophthalmic Surg. 1982;13(9):713-4.

6. Duy TP, Wollensak J. Ciliary block (malignant) glaucoma following posterior chamber lens implantation. Ophthalmic Surg. 1987;18(10):741-4.

7. Reed JE, Thomas JV, Lytle RA, Simmons RJ. Malignant glaucoma induced by an intraocular lens. Ophthalmic Surg. 1990;21(3):177-80.

8. Cashwell LF, Martin TJ. Malignant glaucoma after laser iridotomy. Ophthalmology. 1992;99(5):651-8; discussion 658-9. Comment in Ophthalmology. 1992;99(11):1641-2. Ophthalmology. 1992;99(12):1751-2.

9. Robinson A, Prialnic M, Deutsch D, Savir H. The onset of malignant glaucoma after prophylactic laser iridotomy. Am J Ophthalmol. 1990;110(1):95-6.

10. Brooks AM, Harper CA, Gillies WE. Occurrence of malignant glaucoma after laser iridotomy. Br J Ophthalmol. 1989;73(8):61720. Comment in Br J Ophthalmol. 1990;74(9):576.

11. Hodes BL. Malignant glaucoma after laser iridotomy. Ophthalmology. 1992;99(11):1641-2. Comment on Ophthalmology. 1992;99(5):651-8; discussion 658-9.

12. Rieser JC, Schwartz B. Miotic-induced malignant glaucoma. Arch Ophthalmol. 1972;87(6):706-12.

13. Hardten DR, Brown JD. Malignant glaucoma after Nd-YAG cyclophotocoagulation. Am J Ophthalmol. 1991;111(2):245-7.

14. Cashwell LF, Martin TJ, Winston-Salem. Malignant glaucoma developing after laser sclerostomy. Ophthalmology. 1991;98 (Suppl):161.

15. Ozeki N, Yuki K, Kimura I. Alternative approach to treating malignant glaucoma after trabeculectomy with unplanned zonulectomy. Clin Ophthalmol. 2010;4:383-5.

16. Salvetat ML, Zeppieri M, Tosoni C, Brusini P. Comparisons between Pascal dynamic contour tonometry, the TonoPen, and Goldmann applanation tonometry in patients with glaucoma. Acta Ophthalmol Scand. 2007;85(3):272-9. 
17. Labiris G, Gkika M, Katsanos A, Fanariotis M, Alvanos E, Kozobolis V. Anterior chamber volume measurements with Visante optical coherence tomography and Pentacam: repeatability and level of agreement. Clin Experiment Ophthalmol. 2009;37(8):772-4.

18. Herschler J. Laser shrinkage of the ciliary processes. A treatment for malignant (ciliary block) glaucoma. Ophthalmology. 1980;87(11):1155-9.

19. Lee PF, Shihab Z, Eberle M. Partial ciliary process laser photocoagulation in the management of glaucoma. Lasers Surg Med. 1980;1(1):85-92.

20. Epstein DL, Steinert RF, Puliafito CA. Neodymium-YAG laser therapy to the anterior hyaloid in aphakic malignant (ciliovitreal block) glaucoma. Am J Ophthalmol. 1984;98(2):137-43.

21. Melamed S, Ashkenazi I, Blumenthal M. Nd-YAG laser hyaloidotomy for malignant glaucoma following one-piece $7 \mathrm{~mm}$ intraocular lens implantation. Br J Ophthalmol. 1991;75(8):501-3.

22. Brown RH, Lynch MG, Tearse JE, Nunn RD. Neodymium-YAG vitreous surgery for phakic and pseudophakic malignant glaucoma. Arch Ophthalmol. 1986;104(10):1464-6.
23. Little BC, Hitchings RA. Pseudophakic malignant glaucoma: Nd:YAG capsulotomy as a primary treatment. Eye (Lond). 1993; 7(Pt 1):102-4.

24. Weiss H, Shin DH, Kollarits CR. Vitrectomy for malignant (ciliary block) glaucomas. Int Ophthalmol Clin. 1981;21(1):113-9.

25. Lynch MG, Brown RH, Michels RG, Pollack IP, Stark WJ. Surgical vitrectomy for pseudophakic malignant glaucoma. Am J Ophthalmol. 1986;102(2):149-53.

26. Byrnes GA, Leen MM, Wong TP, Benson WE. Vitrectomy for ciliary block (malignant) glaucoma. Ophthalmology. 1995;102(9):1308-11.

Autor correspondente:

Marcos Antônio Ferraz Rego

Centro Brasileiro da Visão

Avenida L2 Sul - Quadra 613 - Lote 91 - CEP 70200-730 -

Brasília (DF), Brasil

Tel: (61)3547-3441 / Fax: (61) 3214-5151 